\title{
Eric Voegelin e i simboli del politico
}

\author{
di Massimo Mezzanzanica
}

massimo.mezzanzanica@gmail.com

\begin{abstract}
The article deals with Eric Voegelin's analysis of the symbolic dimension of politics and history. It focuses at first on the analysis of the relationship between religion, politics and totalitarianism in the essay on "political religions" and outlines some aspects of the context in which this essay was written. Some characteristics of Voegelin's methodological reflections on the idea of a "new science" of politics are then presented and it is then shown how the attempt to understand the relationship between ideas and symbols opens a new horizon of research, in which the relationship between symbol and representation, and that between symbol, history and being, around which the monumental, and unfinished work Order and History moves, are the main axes.
\end{abstract}

Keywords: Political Ontology, Philosophical Anthropology, Religion and Politics, Representation, Theory of History

\section{Introduzione: il politico e il simbolico}

La sussistenza di un rapporto fondamentale tra il politico e la dimensione simbolica, o al limite la coincidenza tra questi due ambiti dell'umano, è legata al ruolo costitutivo del simbolico in rapporto alla vita dell'essere umano. Animale "per natura politico» - physei politikon zoon, secondo la definizione aristotelica $^{1}$-, l'uomo è anche, seguendo ancora Aristotele, zoon logon echon, animale capace di parola ${ }^{2}$ e, con le parole di Cassirer, animal symbolicum, che vive nella dimensione del simbolico, ossia in un "terzo sistema" di adattamento all'ambiente, diverso dal sistema ricettivo e da quello reattivo ${ }^{3}$.

\footnotetext{
1 Arist., Politica, 1253a, 2-3. C.A. Viano (edizione Rizzoli 2002, 20177, p. 77) traduce «l'uomo è un animale che per natura deve vivere in una città».

2 Arist., Politica, 1253a, 9-10. Nella tr. di Viano: «l'uomo è l'unico animale che abbia la favella».

${ }^{3}$ E. Cassirer, Saggio sull'uomo. Introduzione ad una filosofia della cultura umana (1944), tr. it. di C. D’Altavilla, Armando Editore, Roma 1968, p. 81: «La ragione è un termine poco
} 
$\mathrm{Al}$ di là dei diversi modi in cui è possibile intendere la nozione di simbolo ${ }^{4}$, la convivenza tra gli esseri umani, che porta a costruire istituzioni sociali e politiche, forme di governo della vita e strutture di potere, non può prescindere dall'articolazione linguistica e simbolica che caratterizza ogni ambito della vita dell'umano. Da questo punto di vista, la questione del simbolico sembra svolgere un ruolo fondamentale in rapporto a due temi: quello di un'ontologia del politico in quanto dimensione distinta dalla politica $^{5}$, e quello dell'istituzione in quanto elemento centrale e costitutivo del politico ${ }^{6}$. Per chiarire in prima approssimazione il senso della distinzione politica/politico e la sua relazione col piano dell'istituzione e del simbolico ci si può per esempio richiamare a Claude Lefort, che ha criticato l'impostazione "positivistica" che a suo avviso caratterizza l'analisi delle scienze sociali, e si è richiamato alla tradizione alternativa della filosofia politica ${ }^{7}$, formulando su queste basi in modo pregnante la distinzione tra lo studio della politica, intesa come l'ambito delle istituzioni politiche, sociali ed economiche effettivamente esistenti, ovvero il piano istituito dell'esercizio del potere, e la riflessione sul politico, che presuppone il processo istituente della messa in

adeguato se si vuole abbracciare in tutta la loro ricchezza e varietà le forme della vita culturale dell'uomo. Queste forme sono essenzialmente forme simboliche. Invece di definire l'uomo come animal rationale si dovrebbe dunque definirlo come un animal symbolicum». Sul sistema simbolico come "terzo sistema" cfr. ivi, p. 79.

${ }^{4}$ Gilbert Durand, L'imagination symbolique, Presses Universitaires de France, Paris 1964, oppone per esempio le ermeneutiche "instaurative" (Jung, Bachelard, Ricoeur), che distinguono il simbolo dal segno e considerano il mondo simbolico nella sua specificità, e le ermeneutiche "riduttive" (Freud, Dumézil, Lévi-Strauss), che tendono a ridurre, in modo intellettualistico, il simbolo al segno. Secondo Durand, la posizione di Cassirer si situa in una zona intermedia tra queste due impostazioni.

${ }^{5}$ Sulla distinzione tra la politica, come insieme di procedure e processi decisionali attinenti questioni fattuali, e il politico, in quanto dimensione di costituzione della società che ha a che fare con questioni di senso, cfr. gli interventi raccolti in T. Bedorf, K. Röttgers (hrsg. v.), Das Politische und die Politik, Suhrkamp, Frankfurt am Main 2010, e in particolare il contributo di T. Bedorf, "Das Politische und die Politik. Konturen einer Differenz", ivi, p. 13-37.

${ }_{6}$ Sul ruolo dell'istituzione in rapporto all'ontologia politica cfr. R. Esposito, Pensiero istituente. Tre paradigmi di ontologia politica, Einaudi, Torino 2020.

7 C. Lefort, La questione della democrazia, in C. Lefort, Saggi sul politico. XIX-XX secolo (1986), tr. it. di B. Magni e P. Montanari, Il Ponte, Bologna 2007, p. 19: «Ripensare il politico implica una rottura con il punto di vista della scienza in generale, e in particolare con il punto di vista che si è imposto nelle cosiddette scienze politiche e nella sociologia politica». 
forma (mise-en-forme) dei rapporti sociali. Questa messa in forma, che ha il duplice significato di un conferimento di senso (mise-en-sens) e di una messa in scena (mise-en-scène), consente di accedere all'essere del politico, che resta precluso a un approccio obiettivistico alla politica, il quale si ferma alle singole forme storiche della costituzione politica senza interrogarne i presupposti ${ }^{8}$. In quanto conferimento di senso, il politico è caratterizzato da una struttura semantica che dà un ordine alla società rendendolo accessibile alla percezione degli individui che la compongono; in quanto messa in scena, la forma politica si dà una rappresentazione di sé senza la quale non potrebbe esistere ${ }^{9}$. Per riferirsi a sé stessa sul piano operativo e istituzionale, una società deve istituirsi come unità, deve cioè creare un ambito comune del politico al cui interno possa aver luogo la differenziazione sociale, e questo processo si svolge sul piano simbolico. Questo vuol dire che il simbolico è un elemento centrale della costituzione del reale, e di quell'aspetto fondamentale del reale per la vita dell'essere umano che è la realtà politica. La vita umana, come ha scritto Pierre Legendre riprendendo il vitam instituere di Marciano, va istituita, e il mezzo di questa istituzione è la dimensione "finzionale" del simbolico"10. Quest'ultimo può essere inteso nel senso della fictio come "figura veritatis" e come imitazione della natura di cui ha parlato Ernst Kantorowicz, altro eminente studioso che ha indagato gli aspetti simbolici e linguistici del politico e della storia, riferendosi a Tommaso d'Aquino e alla concezione del diritto dei glossatori e dei commentatori medievali del diritto romano ${ }^{11}$.

\footnotetext{
${ }^{8}$ Come sottolinea Roberto Esposito, Pensiero istituente, cit., p. 158, Lefort ha espresso alcune riserve anche nei confronti della filosofia politica che, sia pure in un'ottica meno specialistica di quella delle scienze sociali, mostrava la tendenza a "adoperare determinate categorie politiche senza discuterne preliminarmente i presupposti», rivelando così un «deficit ermeneutico» analogo a quello delle scienze sociali.

${ }^{9}$ C. Lefort, Permanenza del teologico-politico?, in C. Lefort, Saggi sul politico, cit., p. 262.

${ }^{10}$ P. Legendre, Antropologia dogmatica. Definizione di un concetto (1996), tr. it. a cura di L. Avitabile, Giappichelli, Torino 2001.

11 E. Kantorowicz, "La sovranità dell'artista" (1961), in E. Kantorowicz, La sovranità dell'artista. Mito e immagine tra Medioevo e Rinascimento, a cura di M. Ghelardi, tr. it. di M. Balli, Marsilio, Venezia 1985, pp. 21-22.
} 
Tra i pensatori che nel Novecento si sono dedicati all'analisi del simbolico in rapporto alla dimensione politica e, in senso più ampio, in rapporto alla comprensione della storicità della vita dell'uomo, spicca la figura di Eric Voegelin. Al di là delle differenze rispetto alla prospettiva di Lefort, anche nell'opera di Voegelin l'interesse per gli aspetti simbolici del politico è legato a un movimento di allontanamento dall'approccio alla politica caratteristico delle scienze sociali. Anche Voegelin oppone in modo sempre più articolato e radicale al "positivismo" delle scienze sociali e giuridiche un approccio filosofico alla politica basato da una parte sull'analisi dei simboli in cui si esprimono le esperienze umane, e tra esse le esperienze dell'ordine politico, e dall'altra sul ritorno, nelle mutate condizioni della modernità, all'impostazione della politikē epistēmē inaugurata nell'antica Grecia da Platone e Aristotele. Questo approccio trova la sua formulazione metodica compiuta, e una sua prima realizzazione, nell'opera del 1952 The New Science of Politics, e si realizza poi, almeno parzialmente, nella monumentale e incompiuta opera Order and History, i cui primi tre volumi vengono pubblicati tra il 1956 e il 1957, mentre il quarto appare nel 1974 e il quinto, postumo, nel 1985. Un primo momento di cristallizzazione di questa impostazione è costituito dal breve ma denso e importante saggio del 1938 Die politischen Religionen. A questo saggio, e al contesto in cui esso matura e prende forma, è dedicata la prima parte del nostro contributo, mentre nel seguito, riferendoci a The New Science of Politics e ad alcune parti di Order and History, considereremo sia le riflessioni metodologiche voegeliniane sia alcuni aspetti della sua analisi dei simboli politici e delle esperienze dell'ordine e cercheremo di mostrare come tale analisi si collochi in una prospettiva di carattere ontologico. 


\section{Religione e politica: tra antropologia filosofica e storia delle idee}

Nell'opera di Voegelin, il saggio Die politischen Religionen costituisce al tempo stesso un punto di arrivo e un momento di transizione a una nuova prospettiva di ricerca ${ }^{12}$. Quando esce la prima edizione del saggio, Voegelin, giovane Privatdozent di Teoria del diritto e Sociologia formatosi alla scuola di Hans Kelsen e di Othmar Spann, ha alle spalle alcuni studi nei quali è maturato il suo distacco dall'impostazione positivistica di Kelsen ${ }^{13}$. Rievocando i risultati di questi studi nelle Autobiographical Reflections, Voegelin evidenzia i limiti formalistici di un approccio esclusivamente giuridico allo Stato: identificando lo Stato col diritto, Kelsen trasforma lo Stato in un concetto giuridico e non riesce a cogliere $\mathrm{i}$ processi di identificazione profondi che si trovano alla base della realtà costituzionale e che le danno concretezza. Per Voegelin si tratta invece di riconoscere, da una parte, che la realtà dello Stato non è riducibile alla «pura forma logica», in quanto «lo Stato non è una sfera scientifica», e, dall'altra, che tale realtà risponde a una logica di tipo «simbolico» 14 .

Alla fine degli anni Venti, questa concezione si concretizza nel progetto di una Allgemeine Staatslehre orientata in senso antipositivistico, di cui Voegelin conclude le prime due parti, ma non la terza, che doveva essere dedicata a una teoria del mito, di cui incomincia ad avvertire l'importanza ${ }^{15}$. Nel corso degli anni Trenta, i temi del mito e del rapporto tra religione e politica emergono anche nei due libri sull'idea di razza, elogiati più tardi da Hannah Arendt come la «migliore esposizione del pensiero razziale dal punto

\footnotetext{
${ }^{12} \mathrm{Su}$ questa duplice posizione cfr. la Presentazione di S. Chignola, in E. Voegelin, La politica dai simboli alle esperienze, a cura di S. Chignola, Giuffré, Milano 1993.

13 Si tratta dei saggi Reine Rechtslehre und Staatslehre (1924) e di Zur Lehre von der Staatsform (1927).

14 E. Voegelin, Riflessioni autobiografiche (1973), tr. it. di S. Chignola, in E. Voegelin, La politica dai simboli alle esperienze, cit., pp. 95-97.

${ }^{15}$ Cfr. P. J. Opitz, "Ordine e storia. Concezione e sviluppo dell'opera", in E. Voegelin, Ordine e storia, vol I: Israele e la rivelazione, a cura di N. Scotti Muth, tr. it. di G. Rigamonti, Vita e Pensiero, Milano 2009, p. xxx.
} 
di vista della "storia delle idee"»16. Qui, assumendo una posizione dalle implicazioni politiche inequivocabili, Voegelin critica le pretese di fondare l'antropologia in modo naturalistico, evidenziando i limiti di ogni forma di riduzionismo: «È tremendo pensare di dover riconoscere coloro dai quali discendiamo e coloro di cui ci circondiamo non dal loro aspetto, dalle loro parole e dai loro gesti ma dal loro indice cranico e dalle proporzioni delle loro estremità» 17 .

In una prospettiva consonante con l'antropologia filosofica che in quegli anni andava sviluppandosi - Voegelin cita al riguardo Scheler, Plessner, Groethuysen, Jaspers e Heidegger - il concetto di razza viene ricondotto al rapporto tra corpo e anima e l'idea di corpo viene vista come l'idea fondamentale della teoria dello Stato, poiché le radici dello Stato vanno cercate nell'essenza dell'uomo. Da Scheler Voegelin riprende l'idea di apertura come caratteristica essenziale dell'essere umano, che resterà uno dei tratti costanti della sua concezione antropologica ${ }^{18}$. Attraverso lo studio dell'idea di razza emerge il significato politico delle idee e con esso la prospettiva di una antropologia politica che studia le idee in quanto fenomeni umani e politici considerandole nel loro rapporto con la storia. D'altra parte, nel saggio su Rasse und Staat, richiamandosi a Schelling, Voegelin afferma l'esistenza di un legame tra storia e mito: è attorno al mito che una comunità può articolarsi e trovare la consapevolezza della propria unità e che un popolo può vivere e avere una storia ${ }^{19}$.

16 Si tratta di Rasse und Staat, Mohr, Tübingen 1933 e di Die Rassenidee in der Geistesgeschichte von Ray bis Carus, Junker und Dünnhaupt, Berlin 1933. Del secondo si veda la tr. it. di G. Rossi, Razza. Storia di un`idea, Medusa, Milano 2006, condotta sull'ed. americana The History of the Race Idea: From Ray to Carus, pubblicata a cura di K. Vondung nel vol. III dei Collected Papers di Voegelin, University of Missouri Press, Columbia, Missouri 2000. Cfr. H. Arendt, Le origini del totalitarismo (1966), tr. it. di A. Guadagnin, Einaudi, Torino 2009, p. 222.

${ }^{17}$ E. Voegelin, Razza. Storia di un’idea, cit., pp. 48-49.

18 E. Voegelin, Rasse und Staat, cit., pp. 68-69.

${ }^{19}$ Ivi, pp. 35, 151. Cfr. L. Franco, "Storia e politica nella riflessione di Eric Voegelin", in R. Racinaro (a cura di), Ordine e storia in Eric Voegelin, Edizioni Scientifiche Italiane, Napoli 1988, p. 147. 
Questi studi aprono lo spazio per un'indagine di carattere filosofico del fenomeno religioso, delle sue valenze simboliche, ideologiche e politiche e delle sue implicazioni in rapporto alla formazione della comunità, ed è questo il tema centrale del saggio sulle religioni politiche, in cui Voegelin considera «i movimenti ideologici come variazioni di esperienze religiose» ${ }^{20}$. Questo saggio, scritto da Voegelin poco prima dell'emigrazione negli Stati Uniti successivamente all'Anschluss nazista dell'Austria, è stato per lo più letto come un'analisi delle radici religiose dei movimenti di massa del Novecento e del fenomeno storico del totalitarismo. Anche Voegelin, nella Prefazione al testo, scritta nel 1939 per la seconda edizione uscita a Stoccolma, pur non utilizzando il termine di totalitarismo, sottolinea questo aspetto, dichiarando apertamente la propria «avversione per ogni tipo di collettivismo politico» ${ }^{21}$. Oltre che alle esperienze teoriche già menzionate, la nascita del saggio è legata, come Voegelin ricorda nelle Autobiographical Reflections, all'interesse per le ideologie derivante dagli «stimuli» forniti dalla situazione politica del tempo: le conseguenze della «recente rivoluzione comunista in Russia» e l'«ascesa del fascismo e del nazismo» 22 . È in particolare alla comprensione del nazismo come fenomeno religioso in senso ampio che è dedicata la trattazione. Se si tiene presente però che ai movimenti di massa del Novecento è dedicata solo una parte del penultimo capitolo del testo, e se si considera in che senso Voegelin parli del nazismo come di un fenomeno religioso, si può comprendere la peculiarità della sua impostazione e il significato del suo studio, appartenente al più ampio contesto di un programma di ricerca sulle «implicazioni religiose del pensiero politico» che, come evidenziato da Peter J. Opitz, nasce con gli studi sul problema della razza e si svilupperà fino a Order and History, e costituisce la trattazione parziale di un problema sistematico

\footnotetext{
20 E. Voegelin, Riflessioni autobiografiche, cit., p. 120.

21 E. Voegelin, Le religioni politiche (1938), tr. it. di S. Chignola, in E. Voegelin, La politica dai simboli alle esperienze, cit., p. 21.

22 E. Voegelin, Riflessioni autobiografiche, cit., p. 97.
} 
di più ampia portata: quello del mito politico e dei processi storici in cui nascono, diventano efficaci e muoiono le idee politiche ${ }^{23}$.

A posteriori, Voegelin evidenzia i limiti del suo saggio, consistenti a suo avviso nella vaghezza del concetto di religioni politiche e nell'avere accomunato attraverso questa categoria fenomeni assai diversi tra loro, come la figura del faraone Ekhnaton, l'impero cristiano del medioevo, lo sviluppo degli Stati nazionali, il puritanesimo inglese, il terzo regno di Gioacchino da Fiore, il Leviatano di Hobbes e le filosofie dello Stato di Fichte, Hegel, Marx e Comte $^{24}$. Da questo punto di vista, la ricostruzione di Voegelin sembra condizionata dall'approccio tipologico delle scienze sociali del tempo, da cui pure lo studioso vorrebbe allontanarsi per cogliere i fenomeni politici nella loro storicità. Va però osservato che, al di là di questi limiti, la nozione di religione politica, insieme a nozioni affini come quelle di religione civile o religione secolare, è stata utilizzata in modo fecondo, benché non privo di aspetti controversi, da filosofi, storici e sociologi del Novecento ${ }^{25}$. D'altra parte, attraverso l'analisi delle religioni politiche, Voegelin propone un primo

${ }^{23}$ Cfr. P. J. Opitz, Eric Voegelins Politische Religionen. Kontexte und Kontinuitäten, EricVoegelin-Archiv, München 2006², pp. 5-7, 10.

${ }^{24}$ Cfr. E. Voegelin, Riflessioni autobiografiche, cit., p. 120.

${ }^{25}$ L'approccio ai movimenti politici del Novecento come fenomeni religiosi è centrale nello studio di E. Gentile, Le religioni della politica. Fra democrazie e totalitarismi, Laterza, RomaBari $2007^{2}$, che nel vasto ambito delle "religioni della politica», distingue le due categorie fondamentali di «religione civile», da una parte, e "religione politica» o "religione secolare», dall'altra. Seguendo la ricostruzione dell'uso di questi concetti offerta da Gentile alle pp. 3-7 del suo studio, ricordiamo che, oltre allo storico Karl Polanyi e al teologo Reinhold Niebuhr, che utilizzano il concetto prima di Voegelin (evidenziando nel primo caso «la tendenza del nazionalsocialismo a produrre una religione politica» e applicandolo nel secondo al marxismo e al comunismo), negli anni trenta e quaranta un'analisi delle ideologie totalitarie in termini "religiosi" si trova in Luigi Sturzo, Jacques Maritain e Raymond Aron (in quest'ultimo attraverso il concetto di religione secolare). Negli anni Sessanta il sociologo Robert N. Bellah utilizza il concetto di religione civile nello studio della cultura politica statunitense, riferendosi anche alla nozione voegeliniana (e jaspersiana) di "età assiale", mentre all'inizio degli anni Ottanta Jean-Pierre Sironneau considera le religioni politiche in rapporto al tema della secolarizzazione, prestando attenzione ad aspetti simbolici del nazionalsocialismo e del comunismo come il mito, il rito e la fede. Per le indicazioni bibliografiche in merito, rimandiamo al citato studio di E. Gentile. Sul concetto di religione politica e sulla sua presenza nella discussione contemporanea cfr. anche M. Ley, H. Neisser, G. Weiss, Politische Religion? Politik, Religion und Anthropologie im Werk von Eric Voegelin, Fink, München 2003. 
tentativo di approccio simbolico al politico e individua due temi che resteranno centrali nelle sue opere successive: la politica moderna eredita i contenuti del monoteismo cristiano, i simboli dello Stato sono un surrogato religioso; l'ordine politico è rappresentazione mondana di un ordine trascendente.

In Die politischen Religionen il focus dell'analisi è costituito, più che dal totalitarismo, dalle tendenze profonde della storia europea che lo hanno provocato. Il fenomeno totalitario è visto come la manifestazione di una crisi spirituale dell'Occidente che ha la propria causa «nella secolarizzazione dello spirito, nel distacco di uno spirito - in tal modo soltanto mondano - dalle sue radici nella religiosità 26 .

Per comprendere la nascita e l'affermazione del nazionalsocialismo bisogna quindi affrontare la questione del rapporto tra religione e politica nel contesto della secolarizzazione, un tema affrontato negli stessi anni da Max Weber, che aveva mostrato l'importanza che il fattore religioso, anche in forma secolarizzata, può svolgere nella vita sociale e nella storia. Per trattare questo problema in modo adeguato è però necessario ampliare sia il concetto di religione sia quello di Stato, comprendendo nel concetto di religione «non soltanto le religioni della redenzione, ma anche tutti quegli altri fenomeni che, nel corso dello sviluppo dello Stato, crediamo di poter riconoscere come religiosi» e verificando «se il concetto di Stato non riguardi realmente nient'altro che i rapporti organizzativi-umano-mondani, senza alcuna relazione con la sfera del Religioso» 27 .

Mettendo in discussione la distinzione di competenze tra Stato e religione, Voegelin intende criticare la concezione moderna che considera lo Sato come una sfera profana la cui genesi sarebbe legata a un processo di

${ }^{26}$ E. Voegelin, Le religioni politiche, cit., p. 22.

${ }^{27}$ Ivi, p. 26. 
autolegittimazione ${ }^{28}$. A suo avviso, invece, anche nella modernità la politica implica il riferimento alla dimensione del sacro. La concezione moderna, secondo cui lo Stato è il fondamento della propria sovranità, presuppone la "decapitazione" dell'ordine del creato, con la quale «la testa divina viene troncata ed al posto del Dio trascendente subentra lo Stato quale presupposto ultimo e quale origine del proprio essere» ${ }^{29}$. In questa visione, che secondo Voegelin trova espressione nella concezione hegeliana, lo Stato diventa un potere assoluto, un meccanismo che assorbe in sé la vita del singolo, il quale perde il proprio carattere di «persona particolare» e diventa un mero «membro del tutto» ${ }^{30}$.

Voegelin evidenzia come le religioni politiche si radichino in una dinamica antropologica. Per il suo carattere creaturale e problematico, l'esistenza umana cerca il legame con una realtà sovrapersonale, e questa esperienza può svilupparsi in diversi modi, alcuni più ampi e articolati, fino ad arrivare all'idea di Dio, altri più ristretti e limitati. In questi ultimi casi, l'essere umano attribuisce un carattere sacro a un elemento immediatamente percepito e appartenente al mondo, facendone così un "realissimum» che prende il posto di Dio e che nasconde «tutto il resto: anche, e soprattutto, Dio» ${ }^{31}$.

Da questa dinamica deriva la distinzione tra religioni ultraterrene e religioni intramondane: «le religioni spirituali che trovano il realissimum nel fondamento del mondo debbono chiamarsi per noi religioni ultraterrene (überweltliche); tutte le rimanenti - che trovano il divino in parti del mondo - debbono chiamarsi religioni intramondane (innerweltliche)»32. Tra i simboli sacrali che in questo secondo tipo di religione producono la «unione della sfera

${ }^{28}$ Cfr. S. Chignola, "Filosofia ed esodo. Oltre la teoria politica", in G. Duso (a cura di), Filosofia politica e pratica del pensiero. Eric Voegelin, Leo Strauss, Hannah Arendt, FrancoAngeli, Milano 1988, pp. 69-113.

${ }^{29}$ Ivi, p. 27.

30 Ibidem.

${ }^{31}$ Ivi, p. 30.

32 Ivi, p. 31. 
umana e politica con quella divina», con l'effetto di una divinizzazione dello Stato e della politica, Voegelin, dopo avere descritto la presenza della figura del mediatore nel faraone egizio, ne evidenzia tre: la gerarchia, l'Ecclesia e l'apocalisse, mostrandone la permanenza nel passaggio dal medioevo alla modernità. La gerarchia costituisce «una forma fondamentale di legittimazione del dominio dell'uomo sull'uomo» che «si realizza nel simbolo dell'irradiazione a partire dall'apice divino, attraverso la gerarchia dei signori e degli uffici, sino all'ultimo suddito che ubbidisce» ${ }^{33}$. Il simbolo dell'Ecclesia, che, attraverso un'analogia organica, fa della Chiesa il corpo mistico di Cristo, unisce in sé la gerarchia e la mediazione e costituisce la sostanza che nelle sue trasformazioni determina «le moderne unità politiche intramondane» ${ }^{34}$, anche quelle che sembrerebbero più distanti dal Corpus mysticum del medioevo, come nelle comunità intramondane che riconoscano l'uguaglianza e la fratellanza di tutti i loro membri «e anche là dove comunità e movimenti assumano decisamente posizioni anticlericali ed anticristiane al punto di introdurre una nuova religione di Stato, come nel caso della Rivoluzione francese» ${ }^{35}$. Attraverso l'ulteriore simbolo dell'apocalisse, il medioevo cerca di attribuire un significato interno allo sviluppo storico. Questa interpretazione giunge a piena maturazione nella dottrina del terzo regno di Gioacchino da Fiore, secondo cui al regno divino dell'antica Alleanza succede quello cristiano per arrivare infine al regno dello Spirito Santo, che costituisce un processo di spiritualizzazione grazie al quale gli esseri umani saranno in grado di vivere fraternamente e in povertà, senza alcun ordinamento coercitivo. Questa visione costituisce lo «sfondo storico profondo» a partire da cui si sviluppa la «dinamica apocalittica interna alle religioni politiche moderne» e il suo simbolismo «continua a vivere, nel diciannovesimo e ventesimo secolo, nel simbolismo dei tre regni della filosofia della storia di Marx ed Engels, nel

\footnotetext{
33 Ivi, p. 41.

34 Ivi, p. 44.

35 Ivi, pp. 45-46.
}

Materiali di Estetica - N. 8.2: 2021, Pagina 140 
Terzo Reich nazionalsocialista, nella terza Roma fascista, dopo quella antica e quella cristiana» ${ }^{36}$.

Con la formazione dello Stato moderno nell'età dell'assolutismo l'Ecclesia si suddivide in una molteplicità di comunità particolari e Hobbes è «il grande teologo dell'Ecclesia particolare» ${ }^{37}$. L'aspetto essenziale che Voegelin evidenzia nel simbolo del Leviatano non è il contrattualismo, ma il processo tramite cui la pluralità priva di forma degli esseri umani diventa l'unità del Commonwealth trovando nel sovrano un portatore della propria personalità (Persönlichkeitsträger). Si delinea a questo punto un'analogia tra la teoria hobbesiana e la teoria cristiana del corpus mysticum, tra la Ecclesia intramondana delle nazioni particolari e la Ecclesia di Paolo, poiché il sovrano conferisce unità alla moltitudine nella stessa maniera «simbolico-mistica» in cui lo pneuma conferisce unità all'Ecclesia. Nella concezione di Hobbes, e in genere nel mondo riformato inglese, resta dunque aperto un riferimento alla trascendenza: la teocrazia ebraica si connette con la coscienza nazionale nel simbolo della persona sacrale della comunità, e questo simbolo, in quanto mediatore della volontà di Dio al popolo, mostra tratti simili a quelli della religione imperiale di Ekhnaton. Questo riferimento viene meno negli sviluppi successivi, che portano alla formazione di una comunità compiutamente intramondana, in cui l'Ecclesia «non è più attraversata, in senso sacrale, dalla fonte suprema, ma è essa stessa divenuta l'originaria sostanza sacrale» ${ }^{38}$. Sulla base di una radicale immanentizzazione del sapere, il contenuto del mondo si ingigantisce fino a occultare il mondo e Dio. Nasce una nuova religiosità intramondana, e con essa un nuovo simbolismo, il cui preteso carattere scientifico consiste nell'esclusione di ogni elemento che trascenda i contenuti del mondo. L'«esistenza collettiva intramondana» prende il posto di Dio e la persona diventa uno strumento al servizio del

\footnotetext{
36 Ivi, p. 52.

37 Ivi, p. 53.

38 Ivi, p. 59.
} 
«contenuto sacrale del mondo». "La creazione del mito e la sua propaganda attraverso la stampa e la radio, i discorsi e le cerimonie comunitarie, le adunate e le marce, il lavoro pianificato ed il morire in battaglia, sono le forme intramondane dell'unio mystica» ${ }^{39}$. In seguito alla decapitazione di Dio, la comunità diventa l'unica fonte di legittimazione della persona comunitaria, che si definisce come ordinata dal proprio Persönlichkeitsträger, ovvero dal Führer in cui lo spirito del popolo si esprime compiutamente e irrompe nella realtà storica.

\section{Simbolo, politica e storia}

Dopo il suo arrivo negli Stati Uniti nel 1938, Voegelin riceve la proposta di scrivere una History of Political Ideas. Nel corso degli studi per la realizzazione di quest'opera, le cui dimensioni, col tempo, aumentano molto rispetto a quelle previste inizialmente, Voegelin si trova di fronte a un problema che era rimasto aperto nei suoi studi precedenti, quello del rapporto tra le idee e i simboli. Nel 1945, quando l'opera sembra essere arrivata alla sua conclusione, mentre lavora ai capitoli su Vico e Schelling, Voegelin incontra quella teoria del mito la cui importanza gli si era già palesata nel corso degli anni Trenta. Grazie alla lettura della Philosophie der Mythologie di Schelling, Voegelin si rende conto del fatto che la concezione tradizionale di una «storia delle idee» rappresenta una «deformazione della realtà», a meno che queste idee non si esprimano in "simboli dell'esperienza immediata»" 40 . In seguito a questo mutamento, Voegelin non parlerà più di «idee politiche», ma di «esperienze della realtà» ${ }^{41}$. Da questo momento, al centro delle sue analisi non si trovano più le idee politiche, ma i simboli e i sistemi simbolici attraverso i quali gli esseri umani, nel corso della loro storia, hanno dato

\footnotetext{
39 Ivi, p. 65.

40 E. Voegelin, Riflessioni autobiografiche, cit., p. 131.

41 Ivi, pp. 143-144.
} 
espressione alle loro esperienze. Il passaggio dalla storia delle idee alle esperienze articolate attraverso simboli implicherà una rimodulazione del concetto di storia, che da storia delle idee diventerà la concreta storicità dell'esistenza umana. La necessità di studiare la realtà nelle sue espressioni simboliche porta dunque all'interruzione del lavoro sulla storia delle idee politiche e all'apertura di un nuovo orizzonte di ricerca, in cui il rapporto tra simbolo e rappresentanza, affrontato in The New Science of Politics, e quello tra simbolo, storia e essere, attorno a cui si muove Order and History, costituiscono gli assi portanti. Con questi sviluppi la posizione di Voegelin si precisa nel senso di una filosofia politica di base antropologica e come un'ermeneutica dei simboli ${ }^{42}$.

La "nuova" scienza politica che Voegelin intende costruire si presenta come una restaurazione della consapevolezza dei principi della politike epistēmē degli antichi, in particolare di quella di Platone e di Aristotele ${ }^{43}$, e contiene nella sua denominazione un'allusione alla scienza "nuova" di Vico. Essa risponde all'intenzione di fondare uno studio del mondo umano che faccia valere un'idea di scientificità antitetica rispetto a quella del positivismo, in cui rientra secondo Voegelin anche l'impostazione di Max Weber per la sua concezione relativa al carattere avalutativo della scienza, che esclude dalla considerazione sia i criteri per la selezione dei materiali di studio sia quelli che possono motivare un'etica della responsabilità. Contro l'assunzione dei metodi delle scienze naturali come criteri metodologici validi universalmente, Voegelin fa valere l'idea, di matrice aristotelica, che il metodo debba essere adeguato all'oggetto ${ }^{44}$. A Vico rimanda l'intento di sviluppare una filosofia

\footnotetext{
${ }^{42}$ Cfr. T. Gontier, Voegelin. Symboles du politique, Michalon, Paris 2017.

43 Secondo G. Duso, La rappresentanza politica. Genesi e crisi del concetto, FrancoAngeli, Milano 2003, p. 220, in Voegelin è presente «un'interpretazione che coglie l'unità sostanziale dell'atteggiamento di fondo dei due filosofi»: la filosofia di Platone viene intesa come "cammino dell'anima» e i concetti di Aristotele come simboli, cioè come immagini dell'esperienza dell'anima e non come contenuti di un sapere oggettivo che intenda i termini di sapere e di oggetto in chiave moderna. In questo senso Voegelin parla di una filosofia "platonico-aristotelica».

${ }^{44}$ E. Voegelin, La nuova scienza politica (1952), tr. it. di R. Pavetto, Borla, Roma 1999², p. 37.
} 
antropologica che consideri l'esperienza umana in tutta la sua ampiezza e profondità storica e psichica, come anche l'interesse per le origini dell'umanità, la rivalutazione del linguaggio dei simboli e della retorica, l'affermazione dell'esistenza di un rapporto tra filosofia e mito ${ }^{45}$. A Platone, ma anche a Bergson, rinvia l'idea che l'uomo sia un essere aperto al divino, che la coscienza sia il luogo in cui l'uomo incontra il divino e in cui si fa la storia e che il simbolo esprima la partecipazione dell'uomo all'ordine dell'essere e alla verità. Con le parole di Nicola Matteucci: «L'antropologia, così, si incastona in una più vasta cosmologia, per cui il soggetto conscio può partecipare alla vita del tutto, solo se sa intendere le forze primordiali del cosmo, che si agitano nelle profondità della sua anima» ${ }^{46}$. Tale concezione è espressa in modo icastico dall'incipit di Order and History: «Dio, l'uomo, il mondo e la società formano una comunità d'essere primordiale» 47 .

La categoria di apertura alla realtà, che resta costante nell'antropologia di Voegelin, trova espressione nel concetto platonico di metaxy e in quello di esperienza pura di William James. Entrambi i concetti identificano come esperienza un ambito intermedio (in-between) tra il soggetto e gli oggetti: «L'esperienza non sta né nel soggetto né nel mondo degli oggetti, ma è mediana; ciò significa intermedia tra i poli definiti dall'uomo e dalla realtà di cui egli ha esperienza» ${ }^{48}$. In opposizione a Husserl, il cui modello di coscienza deriva a suo avviso da quello della percezione degli oggetti, e che limita così la filosofia a critica della conoscenza, Voegelin ritiene che l'atteggiamento filosofico consista nel restituire le esperienze di partecipazione a diverse aree della realtà, al di qua della separazione tra soggetto e oggetto, e in questo

\footnotetext{
${ }^{45}$ Cfr. G. Ballacci, "Eric Voegelin e Giambattista Vico. Una lettura retorica", in G.F. Lami (a cura di), Lo stato degli studi voegeliniani. A cinquant'anni dalla pubblicazione di Ordine e storia, FrancoAngeli, Milano 2011, pp. 182-214.

46 N. Matteucci, "Eric Voegelin e il ritorno a Platone", in N. Matteucci, Filosofi politici contemporanei, il Mulino, Bologna 2001, p. 122.

47 E. Voegelin, Ordine e storia, vol. I, cit. p. 15.

48 E. Voegelin, Riflessioni autobiografiche, cit., pp. 137-139.
} 
senso il suo discorso può essere letto come una radicalizzazione della critica mossa da Husserl all'obiettivismo moderno ${ }^{49}$.

In quanto appartiene a una società politica, l'uomo è un essere storico, e questo fa sì che la scienza politica debba essere anche una teoria della storia che ha a che fare con le interpretazioni che l'uomo ha dato di se stesso nella sua vita concreta. La società è un «piccolo cosmo, un cosmion», e in essa risulta centrale la dimensione del significato che gli individui le attribuiscono, un significato che si esprime sempre attraverso simboli, i quali «rendono trasparenti, per il mistero dell'umana esistenza, la struttura interna di codesto cosmion, le relazioni fra i suoi membri e fra i gruppi dei suoi membri, come pure la sua esistenza come un tutto» ${ }^{50}$. L'esperienza dell'ordine, che è ordine politico esistenziale e ordine dell'essere, è costitutiva della società e si esprime attraverso simboli, che possono essere sia quelli prodotti dalla società nei suoi tentativi di autocomprensione sia quelli della scienza. Da qui la centralità del tema della rappresentanza, che costituisce «il problema centrale di una teoria della politica» e che è la "forma attraverso cui una società politica perviene all'esistenza e agisce nella storia» ${ }^{51}$. Se è vero che la realtà dell'esperienza è autointerpretativa, e che le esperienze della realtà possono essere indagate solo passando attraverso la loro espressione nei simboli ${ }^{52}$, altrettanto vero è che la rappresentanza non è solo la dimensione empirica della delega degli interessi, o la dimensione costituzionale dell'organizzazione e della legittimazione del potere, ma, in quanto autorappresentazione di una società, è la condizione dell'esistenza di un ordine politico ${ }^{53}$. Della rappresentanza Voegelin distingue quattro forme, che

\footnotetext{
${ }^{49}$ Cfr. R. Racinaro, "Tempo ed eternità in Eric Voegelin", in R. Racinaro (a cura di), Ordine e storia in Eric Voegelin, cit., p. 171.

${ }^{50}$ E. Voegelin, La nuova scienza politica, cit., p. 61.

${ }^{51}$ Ivi, p. 34.

${ }_{52}$ Cfr. E. Voegelin, Riflessioni autobiografiche, cit., p. 145.

$53 \mathrm{Su}$ questo, e sulla concezione voegeliniana della rappresentanza, cfr. C. Galli, "La rappresentanza, la trascendenza, la storia”, in C. Galli, Contingenza e necessità nella ragione politica moderna, Laterza, Roma-Bari 2009, pp. 211-220.
} 
nella realtà storica sono strettamente intrecciate e che solo l'analisi filosofica può distinguere: alla rappresentanza elementare, legata alle forme rappresentative istituzionali, e alla rappresentanza esistenziale, consistente nella capacità di un governo di realizzare l'idea dell'istituzione, e che è l'elemento che consente a una società di articolarsi e di esistere storicamente, si aggiungono la rappresentanza della verità e la rappresentanza trascendentale. Queste forme della rappresentanza vengono riferite da Voegelin a società e a situazioni storiche concrete. Mentre la rappresentanza elementare è quella che si realizza nelle istituzioni rappresentative liberaldemocratiche, un esempio della rappresentanza esistenziale è dato, secondo Voegelin, dalle istituzioni dell'Urss, che, pur non rappresentando il popolo, rappresentano lo Stato sovietico (questo non significa però che la rappresentanza in senso esistenziale coincida con quella degli Stati totalitari, poiché ogni rappresentanza, secondo Voegelin, è sempre esistenziale, ovvero rappresenta la volontà di agire di una determinata società storica). Se l'idea che viene rappresentata è interamente calata nell'immanenza, come accade nella Cina imperiale o nell'Urss, ovvero in società che ritengono di rappresentare in modo immediato l'ordine cosmico e la verità e dunque si considerano legittimate in modo assoluto, si ha una forma di rappresentanza compatta e tendenzialmente totalitaria. Là dove, invece, come nelle società di Israele, della Grecia antica e del medioevo cristiano, si rompa la compattezza dell'essere che caratterizza le civiltà orientali, si afferma prima una verità "antropologica" e poi una verità "soteriologica", che individua il fondamento della società nella relazione col divino. Seguendo il filo dell'analisi delle diverse forme di rappresentanza e delle diverse articolazioni dell'ordine nella storia, Voegelin evidenzia dunque un passaggio, centrato sulla psiche in quanto "sensorio della trascendenza» 54 , che si produce attraverso un "salto nell'essere» (leap in being, dove il concetto del salto è inteso in senso

${ }^{54}$ E. Voegelin, La nuova scienza politica, cit., p. 108. 
kierkergaardiano), in cui si apre una tensione "noetica" rispetto alla trascendenza e che consente agli uomini di pervenire a una comprensione di sé in quanto collocati nel «dramma dell'essere»55. Questo carattere di apertura e relazione con la trascendenza fa sì che, pur essendo assente dalla storia e non coincidendo con essa, la trascendenza sia «attiva e consaputa proprio in questa tensione» ${ }^{56}$, e questo esclude ogni chiusura totalitaria della società e ogni sua pretesa di identificarsi con la verità. Con le parole di Carlo Galli si può dunque dire che «l'essenza della rappresentanza [...] sembra stare proprio nel fatto che essa contiene in sé questa sorta di 'critica non distruttiva del finito'; che in essa si mostra l'inseparabilità di principio, e al tempo stesso la differenza di principio, fra immanenza e trascendenza, fra politica e idea ${ }^{57}$.

Già dal tardo medioevo, però, si sviluppano tendenze che, in seguito all'abbandono da parte della Chiesa delle prospettive millenaristiche e alla loro sostituzione con l'idea di una salvezza da attendersi alla fine dei tempi, portano a un'immanentizzazione dell'eschaton cristiano e identificano ordine politico, storia e verità, eliminando ogni tensione verso la trascendenza. Esemplare, a questo riguardo, la contrapposizione tra le concezioni della storia di sant'Agostino, secondo cui la città divina e la città terrestre si distingueranno solo col giudizio finale, e di Gioacchino da Fiore, che, applica il simbolo della Trinità al corso della storia, ridivinizzando così la società e creando l'insieme di simboli in base a cui la modernità interpreta la politica e

\footnotetext{
55 E. Voegelin, Ordine e storia, vol. I, cit. pp. 25-26, 15. Sul rapporto con Kierkegaard del concetto di salto nell'essere, cfr. E. Voegelin, Riflessioni autobiografiche, cit., p. 144.

56 C. Galli, Contingenza e necessità nella ragione politica moderna, cit., p. 214.

${ }^{57}$ Ivi, p. 217. Questa concezione della rappresentanza si distingue da quella di Carl Schmitt, di cui Voegelin apprezza il tentativo di trovare una categoria che consenta di comprendere le forme di unità di un popolo, ma critica l'approccio formale e non trascendente che rinchiuderebbe la rappresentanza schmittiana nell'orizzonte dell'immanenza. Cfr. E. Voegelin, "La dottrina della costituzione di Carl Schmitt. Tentativo di analisi costruttiva dei suoi principi teorico-politici" (1931), tr. it. di G. Zanetti, in G. Duso (a cura di), Filosofia politica e pratica del pensiero, cit., pp. 291-314.
} 
la storia ${ }^{58}$. Attraverso i simboli del Terzo regno, del leader, del profeta gnostico e della fratellanza di persone autonome, e attraverso la sua filosofia della storia, che cerca di individuare un eidos, ovvero un significato, della storia, Gioacchino pone le basi delle tendenze filosofiche e politiche che dominano la modernità e che Voegelin definisce ora "gnostiche", tendenze incarnate esemplarmente dalle filosofie di Hegel, Marx, Comte e Nietzsche e dalle diverse forme di progressismo, utopismo e attivismo rivoluzionario da cui nasceranno i totalitarismi del Novecento. Nel quadro di questo nuovo gnosticismo, in cui Voegelin, a partire da The New Science of Politics, ravvisa il carattere di fondo della modernità ${ }^{59}$, si colloca anche il pensiero di Hobbes, che se, per un verso, con la sua teoria della rappresentanza, intende riformulare il problema dell'ordine politico in modo da arginare gli effetti distruttivi dello gnosticismo dei puritani facendo del cristianesimo «una specie di theologia civilis inglese, nel senso varroniano dell'espressione», per l'altro, in quanto fa derivare l'autorità del cristianesimo dalla «ratifica governativa», sembra escludere ogni riferimento alla verità dell'anima e ogni

\footnotetext{
58 Sull'opposizione tra le visioni della storia di sant'Agostino e di Gioacchino da Fiore attira l'attenzione Augusto del Noce nel suo saggio "Eric Voegelin e la critica dell'idea di modernità", in E. Voegelin, La nuova scienza politica, cit., pp. 12-13. Del Noce (ivi, pp. 18, 20) segnala la mancata distinzione da parte di Voegelin tra la gnosi antica e la nuova gnosi: mentre la prima era "posizione di verità", la seconda "sorge per dar soddisfazione a un bisogno pratico", ciò che determina una differenza qualitativa delle due gnosi: «in quanto postcristiana, la nuova gnosi deve attribuire all'uomo il potere creatore». Per una problematizzazione della concezione voegeliniana della gnosi cfr. anche E. Webb, "Voegelin's Gnosticism Reconsidered", The Political Science Reviewer, XXXIV, 2005, pp. 48-76.

${ }_{59}$ Questa linea di pensiero, sviluppata nel quarto, quinto e sesto capitolo di The New Science of Politics, intitolati rispettivamente "Lo gnosticismo caratteristica della modernità", "Rivoluzione gnostica. Il caso puritano" e "La fine della modernità", prosegue nei saggi Wissenschaft, Politik und Gnosis (1959) e Ersatz Religion: The Gnostic Mass Mouvements of Our Time (1959), di cui si veda la tr. it. di A. Munari, in E. Voegelin, Il mito del mondo nuovo. Saggi sui movimenti rivoluzionari del nostro tempo, Rusconi, Milano 1970. Secondo Voegelin, nello gnosticismo moderno, correlativa alla risacralizzazione della società è la tendenza alla divinizzazione dell'uomo. Ricordiamo qui alcune delle ricerche citate da Voegelin in rapporto all'ambito problematico dello gnosticismo: Abendländische Eschatologie, di Jacob Taubes, Meaning in History, di Karl Löwith, Theologie der Geschichte e Prometheus, di Hans Urs von Balthasar, Gnosis und spätantiker Geist, di Hans Jonas, Le drame de l'humanisme athée, di Henri de Lubac, L'Homme révolté, di Albert Camus e The Pursuit of the Millennium, di Norman Cohn.
} 
apertura alla trascendenza, identificando l'ordine politico con l'ordine costituito della società in un modo coerente con la prospettiva immanentistica dello gnosticismo ${ }^{60}$.

In opposizione alle tendenze immanentistiche della modernità, nella visione di Voegelin la coscienza, o l'anima, è al tempo stesso il luogo da cui scaturiscono i problemi dell'ordine umano nella società e nella storia e la dimensione mediana in cui si colloca la tensione tra l'essere temporale dell'uomo e l'essere eterno ${ }^{61}$. Per questo motivo la filosofia della coscienza costituisce il «nucleo essenziale» 62 della filosofia politica e questa, come le ricerche storiche di Voegelin, è volta sia a suscitare una comprensione della struttura dell'ordine del presente e della "pneumopatologia"63 che lo caratterizza, sia al tentativo "inattuale" di riproporre il problema dell'ordine e il valore dell'esperienza della trascendenza nel mondo contemporaneo ${ }^{64}$. Dove il rimando alla trascendenza, e alla sua presenza assente, intende salvaguardare al tempo stesso una visione non dogmatica del rapporto col sacro, un rapporto critico della teoria filosofica con la forma politica e una visione della storia aperta e non totalizzante, perché, lungi dal chiudersi su se stesso nella coincidenza con un eidos, ovvero con un significato predeterminato, «il corso della storia si protende nell'ignoto futuro» 65 .

60 E. Voegelin, La nuova scienza politica, cit., pp. 192-193.

${ }^{61}$ E. Voegelin, Essere eterno nel tempo, tr. it. in R. Racinaro (a cura di), Ordine e storia in Eric Voegelin, cit., p. 190.

62 E. Voegelin, Anamnesis. Teoria della storia e della politica (1966), tr. it. a cura di C. Amirante, Giuffré, Milano 1972, p. 3.

${ }^{63}$ Cfr. G. Parotto, "Pneuma e pneumopatologia nel pensiero di Eric Voegelin", Politica e religione. Annuario di teologia politica, 2010-2011, pp. 233-259.

${ }^{64}$ Per un approfondimento del rapporto fra trascendenza e ordine politico cfr. G. Zanetti, La trascendenza e l'ordine. Saggio su Eric Voegelin, Clueb, Bologna 1989.

65 E. Voegelin, La nuova scienza politica, cit., p. 155. Per una più ampia immagine della concezione voegeliniana della storia rimandiamo ai seguenti studi: E. Webb, Eric Voegelin: Philosopher of History, University of Washington Press, Seattle and London 1981; E. Sandoz, The Voegelinian Revolution. A biographical Introduction, Transaction Pubs., New Brunswick, New Jersey 2000 (in part. i capitoli 4, 5 e 6); Stephen A. McKnight, "The Evolution of Voegelin's Theory of Politics and History", in S. A. McKnight (ed. by), Eric Voegelin's Search for Order in History, Expanded Edition, University Press of America, Lanham, Maryland 1987, pp. 26-45. 


\section{Nota bibliografica}

ARENDT, Hannah, Le origini del totalitarismo (1966), tr. it. di A. Guadagnin, Einaudi, Torino 2009.

ARISTOTELE, Politica, tr. it. di C. A. Viano, Rizzoli, Milano $2017^{7}$.

BALLACCI, Giuseppe, "Eric Voegelin e Giambattista Vico. Una lettura retorica", in G. F. Lami (a cura di), Lo stato degli studi voegeliniani. A cinquant'anni dalla pubblicazione di Ordine e storia, FrancoAngeli, Milano 2011, pp. 182-214.

BEDORF, Thomas, "Das Politische und die Politik. Konturen einer Differenz", in T. Bedorf, K. Röttgers (hrsg. v.), Das Politische und die Politik, Suhrkamp, Frankfurt am Main 2010, pp. 13-37.

CASSIRER, Ernst, Saggio sull'uomo. Introduzione ad una filosofia della cultura umana (1944), tr. it. di C. D’Altavilla, Armando Editore, Roma 1968.

DURAND, Gilbert, L’imagination symbolique, Presses Universitaires de France, Paris 1964.

CHIGNOLA, Sandro, "Filosofia ed esodo. Oltre la teoria politica", in G. Duso (a cura di), Filosofia politica e pratica del pensiero. Eric Voegelin, Leo Strauss, Hannah Arendt, FrancoAngeli, Milano 1988, pp. 69-113.

DEL NOCE, Augusto, "Eric Voegelin e la critica dell'idea di modernità", in E. Voegelin, La nuova scienza politica, tr. it. di R. Pavetto, Borla, Roma 1999², pp. 7-28.

DUSO, Giuseppe, La rappresentanza politica. Genesi e crisi del concetto, FrancoAngeli, Milano 2003. 
ESPOSITO, Roberto, Pensiero istituente. Tre paradigmi di ontologia politica, Einaudi, Torino 2020.

FRANCO, Luigi, "Storia e politica nella riflessione di Eric Voegelin", in R. Racinaro (a cura di), Ordine e storia in Eric Voegelin, Edizioni Scientifiche Italiane, Napoli 1988, pp. 143-153.

GALLI, Carlo, "La rappresentanza, la trascendenza, la storia", in C. Galli, Contingenza e necessità nella ragione politica moderna, Laterza, RomaBari 2009, pp. 209-236.

GENTILE, Emilio, Le religioni della politica. Fra democrazie e totalitarismi, Laterza, Roma-Bari $2007^{2}$.

KANTOROWICZ, Ernst, "La sovranità dell'artista" (1961), in E. Kantorowicz, La sovranità dell'artista. Mito e immagine tra Medioevo e Rinascimento, a cura di M. Ghelardi, tr. it. di M. Balli, Marsilio, Venezia 1985, pp. 17-38.

GONTIER, Thierry, Voegelin. Symboles du politique, Michalon, Paris 2017.

LEFORT, Claude, La questione della democrazia, in C. Lefort, Saggi sul politico. XIX-XX secolo (1986), tr. it. di B. Magni, P. Montanari, Il Ponte, Bologna 2007, pp. 17-31.

—, Permanenza del teologico-politico?, in C. Lefort, Saggi sul politico. XIX-XX secolo (1986), tr. it. di B. Magni, P. Montanari, Il Ponte, Bologna 2007, pp. 255-305.

LEGENDRE, Pierre, Il giurista artista della ragione, tr. it. a cura di L. Avitabile, Giappichelli, Torino 2001.

LEY, Michael, NEISSER, Heinrich, WEISS, Gilbert, Politische Religion? Politik, Religion und Anthropologie im Werk von Eric Voegelin, Fink, München 2003. 
MATTEUCCI, Nicola, "Eric Voegelin e il ritorno a Platone", in N. Matteucci, Filosofi politici contemporanei, il Mulino, Bologna 2001, pp. 115-132.

McKNIGHT, Stephen A., “The Evolution of Voegelin's Theory of Politics and History", in S. A. McKnight (ed. by), Eric Voegelin's Search for Order in History, Expanded Edition, University Press of America, Lanham, Maryland 1987, pp. 26-45.

OPITZ, Peter J., Eric Voegelins Politische Religionen. Kontexte und Kontinuitäten, Eric-Voegelin-Archiv, München $2006^{2}$.

PAROTTO, Giuliana, "Pneuma e pneumopatologia nel pensiero di Eric Voegelin”, Politica e religione. Annuario di teologia politica, 2010-2011, pp. 233-259.

RACINARO, Roberto, "Tempo ed eternità in Eric Voegelin", in R. Racinaro (a cura di), Ordine e storia in Eric Voegelin, Edizioni Scientifiche Italiane, Napoli 1988, pp. 167-180.

SANDOZ, Ellis, The Voegelinian Revolution. A biographical Introduction, Transaction Pubs., New Brunswick, New Jersey 2000.

VOEGELIN, Eric, "La dottrina della costituzione di Carl Schmitt. Tentativo di analisi costruttiva dei suoi principi teorico-politici” (1931), tr. it. di G. Zanetti, in G. Duso (a cura di), Filosofia politica e pratica del pensiero, Eric Voegelin, Leo Strauss, Hannah Arendt, FrancoAngeli, Milano 1988, pp. 291-314.

—, Rasse und Staat, Mohr, Tübingen 1933.

—, Razza. Storia di uñidea (1933), tr. it. a cura di G. Rossi, Medusa, Milano 2006.

—, Le religioni politiche (1938), in E. Voegelin, La politica dai simboli alle esperienze, tr. it. a cura di S. Chignola, Giuffré, Milano 1993, pp. 17-76. 
—, La nuova scienza politica (1952), tr. it. di R. Pavetto, Borla, Roma $1999^{2}$.

—, Ordine e storia, vol. I: Israele e la rivelazione (1956), a cura di N. Scotti Muth, tr. it. di G. Rigamonti, Vita e Pensiero, Milano 2009.

—, Essere eterno nel tempo (1962), tr. it. in R. Racinaro (a cura di), Ordine e storia in Eric Voegelin, Edizioni Scientifiche Italiane, Napoli 1988, pp. 181-206.

—, Anamnesis. Teoria della storia e della politica (1966), tr. it. a cura di C. Amirante, Giuffré, Milano 1972.

—, Il mito del mondo nuovo. Saggi sui movimenti rivoluzionari del nostro tempo, Rusconi, Milano 1970.

-, Riflessioni autobiografiche (1973), in E. Voegelin, La politica dai simboli alle esperienze, tr. it. a cura di S. Chignola, Giuffré, Milano 1993, pp. 77-185.

WEBB, Eugene, Eric Voegelin: Philosopher of History, University of Washington Press, Seattle and London 1981.

—, "Voegelin's Gnosticism Reconsidered", The Political Science Reviewer, XXXIV, 2005, pp. 48-76.

ZANETTI, Gianfrancesco, La trascendenza e l'ordine. Saggio su Eric Voegelin, Clueb, Bologna 1989. 


\section{Nota biografica}

Massimo Mezzanzanica insegna Filosofia e storia in un liceo di Milano e, per l'a.a. 2021-2022, è professore a contratto di Storia e teoria della filosofia presso il Dipartimento di Studi Linguistici e Culturali dell'Università degli Studi di Modena e Reggio Emilia. Tra le sue pubblicazioni: Georg Misch. Dalla filosofia della vita alla logica ermeneutica (FrancoAngeli, Milano 2001), Dilthey filosofo dell'esperienza. Critica della ragione storica: vita, struttura e significatività (FrancoAngeli, Milano 2006), Von Dilthey zu Levinas. Wege im Zwischenbereich von Lebensphilosophie, Neukantianismus und Phänomenologie, (Traugott Bautz, Nordhausen 2012), Corpo, potere e rappresentazione. Figure della sovranità tra teologia politica e antropologia (Mimesis, Milano-Udine 2020). È redattore della rivista «Magazzino di filosofia». 